\title{
Predicting the thermodynamics by using state-dependent interactions
}

\author{
Giuseppe D'Adamo \\ Dipartimento di Scienze Fisiche e Chimiche, \\ Università dell'Aquila, V. Vetoio 10, \\ Loc. Coppito, I-67100 L'Aquila, Italy \\ Andrea Pelissettd \\ Dipartimento di Fisica, Sapienza Università di Roma and INFN, \\ Sezione di Roma I, P.le Aldo Moro 2, I-00185 Roma, Italy \\ Carlo Pierleonis \\ 3 Dipartimento di Scienze Fisiche e Chimiche, \\ Università dell'Aquila and CNISM, UdR dell'Aquila, \\ V. Vetoio 10, Loc. Coppito, I-6r100 L'Aquila, Italy
}

\begin{abstract}
We reconsider the structure-based route to coarse graining in which the coarse-grained model is defined in such a way to reproduce some distributions functions of the original system as accurately as possible. We consider standard expressions for pressure and chemical potential applied to this family of coarse-grained models with density-dependent interactions and show that they only provide approximations to the pressure and chemical potential of the underlying original system. These approximations are then carefully compared in two cases: we consider a generic microscopic system in the low-density regime and polymer solutions under good-solvent conditions. Moreover, we show that the state-dependent potentials depend on the ensemble in which they have been derived. Therefore, care must be used in applying canonical state-dependent potentials to predict phase lines, which is typically performed in other ensembles.
\end{abstract}

PACS numbers: 05.20.Jj, 05.20.Gg, 05.70.Ce, 65.20.De

\footnotetext{
* Address after December 1, 2012: Dip. di Fisica, Sapienza Università di Roma, I-00185 Roma, Italy.
} 


\section{INTRODUCTION}

In condensed matter physics, chemistry, and material science state-dependent interactions arise in many different contexts. They are usually obtained from coarse-graining procedures in which a subset of the degrees of freedom is integrated out (see Refs. 14 for an overview of methods and applications). Indeed, any coarse graining of the original (be it classical or quantum) system induces many-body interactions among the remaining degrees of freedom. The idea is then to replace these complex interactions with state-dependent potentials that are usually taken to be pairwise additive for computational efficiency (if necessary, three-body forces can also be introduced, as in Ref. 5) and that are therefore much more tractable from a theoretical and/or numerical point of view. Different criteria have been used to select the optimal set of state-dependent pair potentials. In the structural route the model with state-dependent interactions is required to reproduce some distribution functions associated with the coarse-grained $(\mathrm{CG})$ degrees of freedom $\underline{-6}^{-14}$ It is also possible to define the CG model by matching the forces $\stackrel{15-17}{\underline{17}}$ acting on the CG sites computed in the original, underlying system, or by requiring the CG model to reproduce solvation free energies. Also state-dependent potentials suitable to treat inhomogeneous systems have been proposed. ${ }^{18,19}$ We should further mention mixed coarse-graining strategies that try to match simultaneously the pair distribution function and some other thermodynamic property, for instance, by constraining the virial pressure to be equal to the pressure of the microscopic model. ${ }^{\underline{1}} \underline{\underline{4}}, \underline{19}$ These mixed approaches, although in principle incorrect since the potential is uniquely defined by the pair distribution function $g(\mathbf{r})$ and the density according to Henderson's theorem, ${ }^{20}$ may still be of value in practical numerical calculations. Indeed, $g(\mathbf{r})$ is only known with statistical errors and is little sensitive to the tail of the potential: visibly different potentials may produce structures with essentially indistinguishable pair distribution functions. $\underline{13}$ Therefore, the large-distance behavior of the CG interactions is determined with a large uncertainty, which might leave some flexibility to implement an additional constraint. We will not discuss these approaches any further, focusing on the conceptual problems of the approach rather than on the difficulties of practical numerical implementations.

In this paper we discuss the structural approach, which dates back to the early days of liquid state theory, $\stackrel{6-10}{=}$ considering state-dependent pair interactions for a very general class of classical systems. The microscopic, underlying model to which the coarse-graining proce- 
dure is applied consists of polyatomic molecules. No hypothesis is made on the nature of the interactions among the atoms: we only require them to be state independent, but, otherwise, they are arbitrary and can, in principle, include any type of many-body terms. Our discussion therefore applies both to monoatomic systems with three- and higher-body interactions (for instance, to noble gases whose accurate study requires the introduction of the threebody Axilrod-Teller potential21 ) and to soft-matter systems, such as polymers, in which the complexity lies in the number of atoms involved rather than in the many-body nature of the atom-atom interactions. $\stackrel{11,14,22}{1}$ Within the CG approach we replace the microscopic system at a given thermodynamic state with a CG system of monoatomic molecules that interact by means of state-dependent pair potentials. The latter are fixed by a structural requirement, the equality of a specific pair distribution function at a given thermodynamic state. If the underlying system is formed by monoatomic molecules, we consider the usual radial pair distribution function. In the case of polyatomic systems, each molecule is replaced by a CG monoatomic molecule located at some point $X$. In many instances, $X$ corresponds to the center of mass of the original molecule, but other choices are possible: for instance, in CG models for star polymers the point $X$ usually coincides with the center of the star $\underline{\underline{22}} \underline{\underline{27}}$ For our purposes, we do not need to specify how $X$ is chosen. We only require the coordinates $\mathbf{r}_{X}$ of $X$ to be a weighted average of the positions of the atoms belonging to the molecule. Once $X$ is chosen, we can consider the $X-X$ pair distribution function

$$
g\left(\mathbf{r}_{1}-\mathbf{r}_{2}\right)=\left\langle\frac{1}{N \rho^{2}} \sum_{i j} \delta\left(\mathbf{r}_{X, i}-\mathbf{r}_{1}\right) \delta\left(\mathbf{r}_{X, j}-\mathbf{r}_{2}\right)\right\rangle,
$$

where $\rho$ is the density and $N$ the number of molecules. The state-dependent potential is fixed by the requirement that the pair distribution function $g_{C G}(\mathbf{r})$ in the CG model is equal to $g(\mathbf{r})$ in the underlying system at the same density. From a practical point of view, the determination of the potential is not an easy task and several method have been devised, like the iterative Boltzmann inversion method $\underline{28}$ and the inverse Monte Carlo method. $\underline{29}, \underline{30}$ Variational approaches have also been discussed, optimizing the coefficients of suitable parametrizations of the CG potentials. $\underline{\underline{31}, 32}$ Note that the structural approach we have described here is not the only one that is used in practical applications. We should also mention the force-matching approach (often called multiscale coarse-graining method ${ }^{15}$ ), in which the state-dependent potential is determined by requiring the $\mathrm{CG}$ system to match the atomistic force on the CG atoms as accurately as possible. Also this method has a 
structural interpretation: the matching condition is equivalent ${ }^{17}$ to require the $\mathrm{CG}$ force to satisfy the appropriate Yvon-Born-Green equation ${ }^{33}$ that relates the pair and the three-body correlation function. We mention that general theoretical formalisms within which different CG approaches can be derived are presented in Refs. 34,35. For a summary of methods and a detailed comparison in a specific example, see Ref. 36.

Once a CG model has been defined, one may use it to predict thermodynamic (and, in the case of more complex CG systems, also structural) properties for the underlying model. For instance, one would like to obtain estimates of the pressure of the microscopic system, by studying (for example, by performing numerical simulations) the simpler CG model.

Structurally derived state-dependent potentials have been mostly discussed in the context of the canonical ensemble, see Refs. $37-40$ and references therein. In this case, the potentials become density and temperature dependent. For this reason, some thermodynamic relations, which can be rigorously proved for systems with state-independent interactions, are no longer satisfied: in particular, the compressibility route and the virial route to the pressure are no longer equivalent for the CG system. This gave rise to several investigations concerning the thermodynamic consistency of models with state-dependent potentials. $37,38,40$ In this paper we again investigate the conceptual issues which arise when considering state-dependent interactions. The first question we wish to address is whether the knowledge of the CG model at density $\rho$ (i.e., of the model with potentials obtained by matching the pair distribution function at density $\rho$ ) allows one to obtain informations on the thermodynamical behavior of the underlying system at the same value of $\rho$. Considering, for instance, pressure and chemical potential, the idea is to compute these two quantities in the CG model. The results of this calculation are then taken as estimates of the pressure and chemical potential of the underlying system. The main problem in implementing this strategy stems from the fact that there is no unambiguous way to define thermodynamic quantities in the presence of statedependent potentials: Approaches that are equivalent for state-independent interactions give different espressions for pressure and chemical potential if potentials depend on the thermodynamic state. Following Ref. 37, we shall consider two different approaches. In the passive approach, the pressure is estimated by using the usual virial expansion, while in the active approach one derives the generalized expression of Ascarelli and Harrison. $\underline{41}$ Both approaches are not thermodynamically consistent and only provide an approximation of the pressure of the underlying model. A quantitative comparison shows that the passive 
approach provides the most accurate estimates, i.e., it best reproduces the pressure of the underlying model. The same result holds for the chemical potential. Widom's method applied to the CG system, even though it does not reproduce the underlying-model value, provides an estimate of the chemical potential that is closest to the underlying-model value than the estimate obtained in the active approach. Note that, if we assume that the densitydependent (DD) potentials are known for all densities $0 \leq \rho \leq \rho_{\max }$ and no phase transitions occur in such density interval, then, via the compressibility route, we can compute the exact equation of state of the underlying model for any $\rho \leq \rho_{\max }$, hence derive the exact pressure, chemical potential, and free energy. However, in this case CG models with DD potentials play little role. Indeed, their computation requires a study of the microscopic model in the whole density interval. But this study would also provide the equation of state directly, without the necessity of introducing any sort of CG model.

It is interesting to extend the analysis of the thermodynamic properties of state-dependent potentials to other ensembles. For instance, for phase-coexistence studies it is more natural to consider the grand-canonical ensemble, while the isothermal-isobaric ensemble may be the best suited to interpret experimental data, since pressure is fixed in experiments. In these ensembles one would consider fugacity- and pressure- dependent pair potentials, respectively. Again, the question we wish to address is how to obtain thermodynamic predictions in these different ensembles by using state-dependent interactions.

The paper is organized as follows. In Sec. [I] we discuss density-dependent potentials and the consistency of several commonly used methods to determine pressure and chemical potential. We show that none of them is exact: none of the thermodynamic quantities extracted via a CG approach is more than an approximate estimate. In Sec. [II] we study the accuracy of the different approximations: first, in Sec. 【IIA we present a general, model-independent discussion in the low-density limit, then, in Sec. IIIB we present a specific calculation for linear polymers under good-solvent conditions. The analysis of Sec. III is extended to the grand-canonical ensemble in Sec. IV, while in Sec. $\mathrm{V}$ we discuss the accuracy with which the thermodynamic behavior is reproduced by using fugacity-dependent potentials. Finally, in Sec. VI we present our conclusions. 


\section{DENSITY-DEPENDENT POTENTIALS IN THE CANONICAL ENSEMBLE}

We start by considering the canonical ensemble and discuss density-dependent interactions. In principle, one should also consider a temperature dependence. However, temperature does not play a role in the discussion (we do not discuss temperature derivatives of the free energy), so that it will be omitted for simplicity. In the structural approach to coarse graining, the basic quantity of interest is the $X-X$ pair distribution function $g(\mathbf{r} ; \rho)$ defined in Eq. (1) for the underlying model. Knowledge of $g(\mathbf{r} ; \rho)$ allows us to obtain the compressibility of the system by using the compressibility relation, ${ }^{33}$ which holds for any choice of the CG site $X$ :

$$
\frac{1}{K(\rho)}=1+\rho \int d^{3} \mathbf{r}[g(\mathbf{r} ; \rho)-1]
$$

where

$$
K(\rho)=\frac{\partial \beta P}{\partial \rho}
$$

is related to the isothermal compressibility $\chi_{T}=\beta /(\rho K)$ and $\beta=1 / k_{B} T$. No other thermodynamic quantity at density $\rho$ can be computed directly from $g(\mathbf{r} ; \rho)$ or $K(\rho)$. However, they can be determined if $K(\rho)$ is known along a thermodynamic path that starts at a state point at which the Helmholtz free energy is known. For instance, in the absence of phase transitions in the density interval $[0, \rho]$, other thermodynamic quantities like pressure, chemical potential, and Helmholtz free energy at density $\rho$ can then be obtained from $K(\rho)$ as

$$
\begin{aligned}
\beta P(\rho) & =\int_{0}^{\rho} d \sigma K(\sigma), \\
\beta \mu(\rho) & =\ln \frac{\rho}{q_{1}}+\int_{0}^{\rho} \frac{d \sigma}{\sigma}(K(\sigma)-1), \\
\frac{\beta F}{N}=\beta f(\rho) & =\beta \mu(\rho)-\frac{\beta P(\rho)}{\rho},
\end{aligned}
$$

where $q_{1}=Z_{1} / V$ and $Z_{1}$ is the partition function of a single, isolated molecule. For convenience, here and in the following we set the de Broglie thermal length equal to one.

In the CG approach we discuss here, one maps each molecule onto a point particle. Under the pair potential approximation, the CG molecules interact by means of the DD potential $V_{D, C G}(\mathbf{r} ; \rho)$, which is defined such as to reproduce the finite-density pair distribution function

$g(\mathbf{r} ; \rho)$. Once $V_{D, C G}(\mathbf{r} ; \rho)$ has been determined, one can use the CG model to compute thermodynamic quantities at the same density $\rho$. In principle one could also use it to study 
the thermodynamic behavior at densities $\rho^{\prime} \neq \rho$, i.e., one could transfer the potentials from one density to the other. The accuracy of this procedure, i.e., the transferability of the potentials, is an important question for CG studies, which, however, will not be considered here.

In order to compute thermodynamic quantities in the CG model, we will consider two different approaches, which, following Ref. 37, will be referred to as the passive and the active approach. To define the two approaches, we consider the more general CG system at fixed interaction potential in the canonical ensemble, i.e. at fixed $V$ and $T$. The Helmholtz free energy is given by

$$
\begin{aligned}
& F_{C G}\left(N, V, T ; \rho_{p}\right)=N f_{C G}\left(\rho ; \rho_{p}\right) \\
& \quad=-k_{B} T \ln \frac{1}{N !} \int d \mathbf{r}_{1} \ldots d \mathbf{r}_{N} e^{-\beta \sum_{i j} V_{D, C G}\left(\mathbf{r}_{i}-\mathbf{r}_{j} ; \rho_{p}\right)}
\end{aligned}
$$

where $\rho=N / V$. The free energy (17) depends on two densities: $\rho=N / V$ is the usual quantity, while $\rho_{p}$ is the density parametrizing the pair potential. No relation between $\rho$ and $\rho_{p}$ is assumed at this stage. Using Eq. (17) we start by defining the pressure and the chemical potential for the general CG model at fixed $\rho_{p}$ :

$$
\begin{gathered}
P_{C G}\left(\rho, \rho_{p}\right)=-\left(\frac{\partial F_{C G}}{\partial V}\right)_{N, T, \rho_{p}}, \\
\mu_{C G}\left(\rho, \rho_{p}\right)=\left(\frac{\partial F_{C G}}{\partial N}\right)_{V, T, \rho_{p}} .
\end{gathered}
$$

If we define

$$
K_{C G}\left(\rho, \rho_{p}\right)=\left(\frac{\partial \beta P_{C G}}{\partial \rho}\right)_{T, \rho_{p}},
$$

we obtain the standard thermodynamic relations

$$
\begin{aligned}
& \beta \mu_{C G}\left(\rho, \rho_{p}\right)=\ln \rho+\int_{0}^{\rho} \frac{d \sigma}{\sigma}\left(K_{C G}\left(\sigma, \rho_{p}\right)-1\right), \\
& \beta P_{C G}\left(\rho, \rho_{p}\right)=\int_{0}^{\rho} d \sigma K_{C G}\left(\sigma, \rho_{p}\right) \\
& \beta f_{C G}\left(\rho, \rho_{p}\right)=\beta \mu_{C G}\left(\rho, \rho_{p}\right)-\frac{\beta P_{C G}\left(\rho, \rho_{p}\right)}{\rho} .
\end{aligned}
$$

These expressions are the analog of those appearing in Eqs. (44), (5), and (6), with $q_{1}=1$, since the CG model we consider consists of monoatomic molecules. Since $\rho_{p}$ is a fixed parameter, the pressure $P_{C G}\left(\rho, \rho_{p}\right)$ and the chemical potential $\mu_{C G}\left(\rho, \rho_{p}\right)$ can be determined 
by using standard methods. In particular, the usual relation between pressure and virial holds. Hence we have ${ }^{33}$

$$
\begin{aligned}
& \beta P_{C G}\left(\rho, \rho_{p}\right)=\beta P_{\mathrm{vir}}\left(\rho, \rho_{p}\right)= \\
& \quad \rho-\frac{2 \pi \beta \rho^{2}}{3} \int_{0}^{\infty} \frac{\partial V_{D, C G}\left(\mathbf{r} ; \rho_{p}\right)}{\partial r} g\left(\mathbf{r} ; \rho, \rho_{p}\right) r^{3} d r .
\end{aligned}
$$

As for the chemical potential, since $\rho_{p}$ is fixed, $\mu_{C G}\left(\rho, \rho_{p}\right)$ can be obtained by using Widom's insertion method 42

$$
\begin{aligned}
& \beta \mu_{C G}\left(\rho, \rho_{p}\right)=\ln \rho- \\
& \ln \left[\frac{1}{V} \int d^{3} \mathbf{r}_{N+1}\left\langle e^{-\beta U_{N+1}\left(\mathbf{r}_{N+1} ; \rho_{p}\right)}\right\rangle_{N, V}\right]
\end{aligned}
$$

where $\langle\cdot\rangle_{N, V}$ is the canonical ensemble average over $N$ molecules in a volume $V, \rho=N / V$, and $U_{N+1}\left(\mathbf{r}_{N+1} ; \rho_{p}\right)$ is the insertion energy of an additional molecule computed by using $V_{D, C G}\left(\mathbf{r} ; \rho_{p}\right)$.

The DD pair potential at density $\rho_{p}=\rho$ is determined by requiring the pair distribution function $g_{C G}(\mathbf{r} ; \rho, \rho)$ to be equal to the $X X$ pair distribution function $g(\mathbf{r} ; \rho)$ in the underlying microscopic model: $g_{C G}(\mathbf{r} ; \rho, \rho)=g(\mathbf{r} ; \rho)$. In the passive approach $\frac{37}{2} \rho_{p}$ is considered as a fixed parameter which is set equal to $\rho$ only at the end of the calculations. Therefore, the pressure for the CG model at density $\rho$ is simply $P_{C G}(\rho, \rho)$; analogously the chemical potential is defined as $\mu_{C G}(\rho, \rho)$. Because of the compressibility relation (2), the CG and the microscopic compressibilities are equal, i.e.

$$
K(\rho)=K_{C G}(\rho, \rho)
$$

This equality does not extend, however, to the other thermodynamic quantities: both $\mu_{C G}(\rho, \rho)$ and $P_{C G}(\rho, \rho)$ differ from the chemical potential and pressure at density $\rho$ of the microscopic model. Indeed, barring unexpected concidences, it is expected in general

$$
\begin{aligned}
& \beta \mu^{\mathrm{exc}}(\rho)=\int_{0}^{\rho} \frac{d \sigma}{\sigma}\left(K_{C G}(\sigma, \sigma)-1\right) \\
& \neq \beta \mu_{C G}^{\mathrm{exc}}(\rho, \rho)=\int_{0}^{\rho} \frac{d \sigma}{\sigma}\left(K_{C G}(\sigma, \rho)-1\right), \\
& \beta P(\rho)=\int_{0}^{\rho} d \sigma K_{C G}(\sigma, \sigma) \\
& \neq \beta P_{C G}(\rho, \rho)=\int_{0}^{\rho} d \sigma K_{C G}(\sigma, \rho),
\end{aligned}
$$


where we used Eq. (16) to replace $K(\rho)$ with $K_{C G}(\rho, \rho)$ in the left-hand side integrals, which give chemical potential and pressure in the underlying model. Hence, if we use the virial route for the pressure or Widom's insertion method for the chemical potential in the CG model, we only obtain approximations to the underlying-model pressure and chemical potential: even with the use of DD potentials, we cannot obtain the underlying-model thermodynamics at density $\rho$ from the study of the CG model at that density. Moreover, the approach is not thermodynamically consistent. Indeed, Eq. (18) implies immediately that the density derivative of $P_{C G}(\rho, \rho)$ differs from $K_{C G}(\rho, \rho)$. Of course, the pressure and chemical potential of the underlying system can be obtained by using $K(\rho)=K_{C G}(\rho, \rho)$ and relations (41) and (5) $\cdot 38$ But this approach essentially uses the equation of state of the microscopic model and does not make use of the CG model with DD potentials.

It is also possible to derive expressions for the CG pressure and chemical potential by using the active approach of Ref. 37. The idea is to start from Eq. (7), set $\rho_{p}=\rho=N / V$, and then take the derivative with respect to $V$. This gives the well-known Ascarelli-Harrison ${ }^{41}$ expression for the pressure:

$$
\begin{aligned}
P_{A H}(\rho)= & \rho^{2} \frac{\partial f_{C G}(\rho, \rho)}{\partial \rho} \\
= & P_{C G}(\rho, \rho)+\rho^{2}\left(\frac{\partial f_{C G}\left(\rho, \rho_{p}\right)}{\partial \rho_{p}}\right)_{\rho_{p}=\rho} \\
= & P_{\operatorname{vir}}(\rho, \rho)+ \\
& \quad 2 \pi \rho^{3} \int_{0}^{\infty} r^{2} d r \frac{\partial V_{D, C G}(\mathbf{r} ; \rho)}{\partial \rho} g_{C G}(\mathbf{r} ; \rho, \rho),
\end{aligned}
$$

where $P_{\text {vir }}(\rho, \rho)$ is explicitly given in Eq. (14). Simple algebra allows us to rewrite

$$
\beta P_{A H}(\rho)=\int_{0}^{\rho} d \sigma\left[K_{C G}(\sigma, \rho)+\frac{\rho(\rho-\sigma)}{\sigma} \frac{\partial K_{C G}(\sigma, \rho)}{\partial \rho}\right],
$$

which shows that $P_{A H}(\rho)$ differs in general from the pressure of the underlying model and that its density derivative differs from the CG quantity $K_{C G}(\rho, \rho)$.

The Ascarelli-Harrison prescription can also be applied to the chemical potential, defining

$$
\begin{aligned}
\mu_{A H}(\rho)= & f_{C G}(\rho, \rho)+\rho \frac{\partial f_{C G}(\rho, \rho)}{\partial \rho}= \\
= & \mu_{C G}(\rho, \rho)+\rho\left(\frac{\partial f_{C G}\left(\rho, \rho_{p}\right)}{\partial \rho_{p}}\right)_{\rho_{p}=\rho}= \\
= & \mu_{C G}(\rho, \rho)+ \\
& 2 \pi \rho^{2} \int_{0}^{\infty} r^{2} d r \frac{\partial V_{D, C G}(\mathbf{r} ; \rho)}{\partial \rho} g_{C G}(\mathbf{r} ; \rho, \rho) .
\end{aligned}
$$


Again, it is easy to verify that such an expression differs from the underlying-model result $\beta \mu^{\mathrm{exc}}(\rho)$. Indeed, we have

$$
\beta \mu_{A H}^{\mathrm{exc}}(\rho)=\int_{0}^{\rho} \frac{d \sigma}{\sigma}\left[K_{C G}(\sigma, \rho)-1+(\rho-\sigma) \frac{\partial K_{C G}(\sigma, \rho)}{\partial \rho}\right] .
$$

As we have seen, the passive and the active approaches provide two different expressions for the pressure. It is interesting to reinterpret these results as follows. For standard thermodynamic systems there are two equivalent approaches to the pressure. One can use the force route, in which one defines the pressure as the force per unit area exerted on the walls of the box containing the system. For a system of $N$ molecules in a volume $V$ interacting with a pair potential $v(\mathbf{r})$, Clausius theorem allows one to relate the pressure $P$ so defined to the virial: $: \underline{33}$

$$
P V=N k_{B} T-\frac{1}{3}\left\langle\sum_{i<j} \mathbf{r}_{i j} \cdot \frac{\partial v\left(\mathbf{r}_{i j}\right)}{\mathbf{r}_{i j}}\right\rangle .
$$

Alternatively, one can define the pressure $P$ by using the work $d W$ necessary to change the volume of the box by an infinitesimal amount $d V, d W=-P d V$, which provides the usual expressions of $P$ as the derivative of the thermodynamic potentials with respect to the volume. The two definitions are obviously equivalent for density-independent potentials but differ in the presence of DD interactions. In the passive approach the pressure satisfies Clausius theorem. This choice looks very natural, since the Clausius equation (23) is a simple mechanical relation that is proved by balancing the forces for a system of a fixed number of particles in a box of fixed volume, hence at fixed density. The density dependence of the potential is irrelevant and therefore, one would naturally expect Eq. (23) to hold also in the presence of DD interactions. On the other hand, $P_{A H}(\rho)$ is defined by using the thermodynamic route, i.e. it satisfies the usual thermodynamic relation $d W=-P_{A H} d V$. However, it is not consistent with Clausius theorem, hence the pressure computed in the active approach is not directly related to the force exterted by the particles. This is due to the fact that the work done when changing the volume of the system has two contributions. One of them corresponds to the work done against the forces acting on the box boundary (this is the only contribution taken into account when considering the virial pressure), the other one takes into account the change of the potential as the volume $V$ is changed at fixed number of particles. From this viewpoint, the active approach certainly looks less natural than the passive one. 


\section{COMPARING THE DIFFERENT DEFINITIONS OF THE PRESSURE AND CHEMICAL POTENTIAL IN THE CANONICAL ENSEMBLE}

As we have seen in the previous section, the DD quantities $P_{C G}(\rho, \rho)$ and $\mu_{C G}(\rho, \rho)$ (passive approach) or their counterparts $P_{A H}(\rho)$ and $\mu_{A H}(\rho)$ only provide approximations to the correct result. In this section we wish to compare them with the exact result, determining quantitatively the size of the discrepancy. In the context of soft-matter systems, $\underline{22}, \underline{43}-\underline{46}$ it is also common to use CG models based on zero-density potentials, i.e. potentials obtained by matching the pair distribution function in the limit $\rho \rightarrow 0$. In this approach the potentials are fixed and then used to obtain predictions at densities $\rho>0$. Hence, in the notation of the previous section, the pressure and the chemical potential correspond to $P_{C G}(\rho, 0)$ and $\mu_{C G}(\rho, 0)$, respectively. These quantities will be compared with the corresponding DD quantities, in order to understand which method provides the best approximation. We shall first consider the low-density limit, which can be analyzed in a model-independent way, and then we shall apply all formulae to a specific soft-matter example, polymers in the semidilute regime. In both cases, the results of the different approaches will be compared with those obtained in the microscopic model.

\section{A. Low-density behavior}

Let us consider the low-density limit. For $\rho \rightarrow 0$ the $X X$ pair distribution function can be expanded as

$$
g(\mathbf{r} ; \rho)=g_{0}(\mathbf{r})+\rho g_{1}(\mathbf{r})+O\left(\rho^{2}\right)
$$

We define $h_{0}(\mathbf{r})=g_{0}(\mathbf{r})-1$,

$$
\hat{g}_{1}(\mathbf{r})=g_{0}(\mathbf{r}) \int d^{3} \mathbf{s} h_{0}(\mathbf{s}) h_{0}(\mathbf{r}-\mathbf{s}),
$$

and $\Delta(\mathbf{r})=g_{1}(\mathbf{r})-\hat{g}_{1}(\mathbf{r})$. The quantity $\Delta(\mathbf{r})$ encodes the contributions of the three-body interactions: for monoatomic systems interacting by means of pairwise additive interactions we have $\Delta(\mathbf{r})=0$ and $g_{1}(\mathbf{r})=\hat{g}_{1}(\mathbf{r})$. Using the compressibility relation (2) and Eq. (24), we can compute $K(\rho)$ in the underlying microscopic model:

$$
K(\rho)=1-\rho I_{0}-\rho^{2}\left(I_{1}+I_{2}\right)+O\left(\rho^{3}\right),
$$


with

$$
\begin{aligned}
I_{0} & =\int d^{3} \mathbf{r} h_{0}(\mathbf{r}), \\
I_{1} & =\int d^{3} \mathbf{r} d^{3} \mathbf{s} h_{0}(\mathbf{r}) h_{0}(\mathbf{s}) h_{0}(\mathbf{r}-\mathbf{s}), \\
I_{2} & =\int d^{3} \mathbf{r} \Delta(\mathbf{r}) .
\end{aligned}
$$

Eqs. (41) and (5) give

$$
\begin{aligned}
\beta P(\rho) & =\rho-\frac{1}{2} \rho^{2} I_{0}-\frac{1}{3} \rho^{3}\left(I_{1}+I_{2}\right)+O\left(\rho^{4}\right), \\
\beta \mu^{\operatorname{exc}}(\rho) & =-I_{0} \rho-\frac{1}{2} \rho^{2}\left(I_{1}+I_{2}\right)+O\left(\rho^{3}\right) .
\end{aligned}
$$

Let us now consider the CG model. First, we need to compute the DD potential $V_{D, C G}\left(\mathbf{r} ; \rho_{p}\right)$. Since we are interested in the low-density limit, we expand it as

$$
V_{D, C G}\left(\mathbf{r} ; \rho_{p}\right)=V_{0 C G}(\mathbf{r})+\rho_{p} V_{1 C G}(\mathbf{r})+O\left(\rho_{p}^{2}\right)
$$

At leading order in the density, we have

$$
g_{C G}\left(\mathbf{r} ; \rho, \rho_{p}\right)=e^{-\beta V_{0 C G}(\mathbf{r})}+O\left(\rho, \rho_{p}\right) .
$$

By requiring the equality of the pair distribution functions, i.e. $g(\mathbf{r} ; \rho)=g_{C G}(\mathbf{r} ; \rho, \rho)$, we obtain

$$
\beta V_{0 C G}(\mathbf{r})=-\ln g_{0}(\mathbf{r})
$$

If we include the first density correction we have

$$
\begin{aligned}
& g_{C G}\left(\mathbf{r} ; \rho, \rho_{p}\right)=e^{-\beta V_{D, C G}\left(\mathbf{r} ; \rho_{p}\right)}[1+ \\
& \left.\quad \rho \int d^{3} \mathbf{s} f\left(\mathbf{s} ; \rho_{p}\right) f\left(\mathbf{s}-\mathbf{r} ; \rho_{p}\right)\right]+O\left(\rho^{2}\right),
\end{aligned}
$$

where

$$
f\left(\mathbf{s} ; \rho_{p}\right)=e^{-\beta V_{D, C G}\left(\mathbf{s} ; \rho_{p}\right)}-1 .
$$

Expanding in $\rho_{p}$, using Eq. (34) and $h_{0}(\mathbf{r})=g_{0}(\mathbf{r})-1$, we obtain

$$
\begin{aligned}
& g_{C G}\left(\mathbf{r} ; \rho, \rho_{p}\right)=g_{0}(\mathbf{r})\left[1-\rho_{p} \beta V_{1 C G}(\mathbf{r})\right] \\
& \quad+\rho g_{0}(\mathbf{r}) \int d^{3} \mathbf{s} h_{0}(\mathbf{s}) h_{0}(\mathbf{r}-\mathbf{s})+O\left(\rho^{2}, \rho \rho_{p}, \rho_{p}^{2}\right) .
\end{aligned}
$$


By requiring $g(\mathbf{r} ; \rho)=g_{C G}(\mathbf{r} ; \rho, \rho)$, we obtain the next correction:

$$
\beta V_{1 C G}(\mathbf{r})=-\frac{\Delta(\mathbf{r})}{g_{0}(\mathbf{r})}
$$

Note that these definitions imply

$$
\int d^{3} \mathbf{r} f\left(\mathbf{r} ; \rho_{p}\right)=I_{0}+\rho_{p} I_{2}+O\left(\rho_{p}^{2}\right)
$$

for $\rho_{p} \rightarrow 0$. We can now compute $K\left(\rho, \rho_{p}\right)$. In the limit $\rho \rightarrow 0$, using the standard expressions valid for a monoatomic system,, 33 we obtain

$$
\begin{aligned}
& K_{C G}\left(\rho, \rho_{p}\right)=1-\rho \int d^{3} \mathbf{r} f\left(\mathbf{r} ; \rho_{p}\right) \\
& \quad-\rho^{2} \int d^{3} \mathbf{r} d^{3} \mathbf{s} f\left(\mathbf{r} ; \rho_{p}\right) f\left(\mathbf{s} ; \rho_{p}\right) f\left(\mathbf{r}-\mathbf{s} ; \rho_{p}\right)+O\left(\rho^{3}\right) .
\end{aligned}
$$

Expanding in $\rho_{p}$ and using Eq. (38), we obtain

$$
K_{C G}\left(\rho, \rho_{p}\right)=1-\rho I_{0}-\rho^{2} I_{1}-\rho \rho_{p} I_{2}+O\left(\rho^{3}, \rho^{2} \rho_{p}, \rho \rho_{p}^{2}\right) .
$$

For $\rho_{p}=\rho$ this expression coincides with Eq. (26), as expected. Using Eqs. (11), (12), and (13) we obtain

$$
\begin{aligned}
& \beta P_{C G}\left(\rho, \rho_{p}\right)=\rho-\frac{1}{2} \rho^{2} I_{0}-\frac{1}{3} \rho^{3} I_{1}-\frac{1}{2} \rho^{2} \rho_{p} I_{2}, \\
& \beta \mu_{C G}^{\mathrm{exc}}\left(\rho, \rho_{p}\right)=-\rho I_{0}-\frac{1}{2} \rho^{2} I_{1}-\rho \rho_{p} I_{2}, \\
& \beta f_{C G}\left(\rho, \rho_{p}\right)=\ln \rho-1-\frac{1}{2} \rho I_{0}-\frac{1}{6} \rho^{2} I_{1}-\frac{1}{2} \rho \rho_{p} I_{2},
\end{aligned}
$$

disregarding higher-order terms in $\rho$ and $\rho_{p}$. From the expression of the free energy, we obtain the Ascarelli-Harrison expressions for the pressure and chemical potential:

$$
\begin{aligned}
\beta P_{A H}(\rho) & =\beta \rho^{2} \frac{d f_{C G}(\rho, \rho)}{d \rho}=\rho-\frac{1}{2} \rho^{2} I_{0}-\frac{1}{3} \rho^{3} I_{1}-\rho^{3} I_{2}, \\
\beta \mu_{A H}^{\mathrm{exc}}(\rho) & =\beta f_{C G}(\rho, \rho)+\beta \rho \frac{d f_{C G}(\rho, \rho)}{d \rho} \\
& =-\rho I_{0}-\frac{1}{2} \rho^{2} I_{1}-\frac{3}{2} \rho^{2} I_{2} .
\end{aligned}
$$

We can thus compare these expressions with the exact ones valid for the underlying model. For the pressure we have

$$
\begin{aligned}
& \beta P_{C G}(\rho, \rho)-\beta P(\rho)=-\frac{1}{6} \rho^{2} I_{2}, \\
& \beta P_{C G}(\rho, 0)-\beta P(\rho)=\frac{1}{3} \rho^{2} I_{2}, \\
& \beta P_{A H}(\rho)-\beta P(\rho)=-\frac{2}{3} \rho^{2} I_{2} .
\end{aligned}
$$


TABLE I: Compressibility factor $Z=\beta P / \rho$ and excess chemical potential for a polymer CG system at $\Phi=0.8$ and 1.5. The reference polymer $Z(\rho)$ and $\mu^{\operatorname{exc}}(\rho)$ have been obtained by using the equation of state reported in Ref. 49 .

\begin{tabular}{cccccccc}
\hline \hline$\Phi$ & $Z(\rho)$ & $Z_{C G}(\rho, 0)$ & $Z_{C G}(\rho, \rho)$ & $Z_{A H}(\rho)$ & $\beta \mu^{\mathrm{exc}}(\rho)$ & $\beta \mu_{C G}^{\mathrm{exc}}(\rho, 0)$ & $\beta \mu_{C G}^{\mathrm{exc}}(\rho, \rho)$ \\
\hline 0.80 & 2.35 & $2.22402(4)$ & $2.44790(4)$ & $2.51(2)$ & 2.56 & $2.3797(2)$ & $2.8108(3)$ \\
1.50 & 3.90 & $3.39847(4)$ & $4.13973(4)$ & $4.28(5)$ & 5.38 & $4.653(4)$ & $6.0929(4)$ \\
\hline \hline
\end{tabular}

From these results we see that the pressure computed by using the DD potentials provides the best approximation to $P(\rho)$. The AH formula is significantly worse - deviations are four times larger than those for the virial pressure $P_{C G}(\rho, \rho)$-as already noted in Refs. 38,39. By using the zero-density potentials one obtains a result which is worse by a factor of two than the DD result.

We can perform the same comparison for the chemical potential. We find

$$
\begin{aligned}
& \beta \mu_{C G}^{\mathrm{exc}}(\rho, \rho)-\beta \mu^{\mathrm{exc}}(\rho)=-\frac{1}{2} \rho^{2} I_{2}, \\
& \beta \mu_{C G}^{\mathrm{exc}}(\rho, 0)-\beta \mu^{\mathrm{exc}}(\rho)=\frac{1}{2} \rho^{2} I_{2}, \\
& \beta \mu_{A H}^{\mathrm{exc}}(\rho)-\beta \mu^{\operatorname{exc}}(\rho)=-\rho^{2} I_{2} .
\end{aligned}
$$

In this case, the chemical potentials derived by using the DD and the density-independent potentials have the same accuracy. Expression (21) is instead worse than both the one obtained by using DD potentials and the one obtained by using zero-density potentials.

Note that all deviations are proportional to $I_{2}$, which encodes the contributions of the (effective) three-body interactions at this order in $\rho$. Hence, consistency is only possible if the three-body terms do not contribute to the thermodynamics, hence $I_{2}=0$, a result which is not generally true since the free energy of the CG model in a generic CG procedure is never pairwise additive.

\section{B. Polymers in the semidilute regime}

To assess the quality of the different approximations to the pressure and the chemical potential, we study the thermodynamic behavior of a system of linear polymers under goodsolvent conditions. We have extensively studied CG models for this type of systems in 
Refs. 43, 44. For two values of the polymer volume fraction $\Phi$,

$$
\Phi=\frac{4 \pi}{3} \hat{R}_{g}^{3} \rho
$$

where $\hat{R}_{g}$ is the zero-density radius of gyration, namely $\Phi=0.80$ and $\Phi=1.50$, we compute $\frac{47}{4}$ the center-of-mass pair distribution function $g(\mathbf{r} ; \rho)$. Then, by using the method described in Refs. 11,12, we determine the DD potential $V_{D, C G}(\mathbf{r} ; \rho)$, checking that it reproduces the full-monomer value of $K$. This is a strong check of the accuracy with which the potential is determined, since the compressibility is very sensitive to the large-distance behavior of the pair potentials. $\underline{\underline{13}}$ For the zero-density potential we use the accurate expression reported in Ref. 48. The Monte Carlo estimates of the compressibility factor $Z=\beta P / \rho$ and of the chemical potential obtained by using the virial route and Widom's method, respectively, are reported in Table I. Completely consistent results are obtained by using the integral-equation approach and the hypernetted-chain (HNC) closure. In our previous work ${ }^{43}$ we showed that the compressibility factor $Z_{C G}(\rho, 0)$ obtained by using zero-density potentials underestimates the polymer $Z(\rho)$, which, in the low-density limit, implies $I_{2}<0$. Our present results are fully consistent. Differences increase with increasing $\Phi$, as expected: the relative error is $5 \%$ and $13 \%$ for $\Phi=0.8$ and 1.5 , respectively. If one uses the DD potentials the compressibility factor is overestimated, by $4 \%$ and $6 \%$ in the two cases. Therefore, in agreement with the low-density analysis, DD potentials provide more accurate estimates of the pressure and clearly represent an improvement with respect to zero-density potentials, especially for the largest value of $\Phi$. We also compute the pressure by using the Ascarelli-Harrison expression. A precise determination is not easy: indeed, it is difficult to estimate accurately the derivative $\partial V_{D, C G}(\mathbf{r} ; \rho) / \partial \rho$, especially for $r / R_{g} \gtrsim 2$. Hence, the additional term that appears in the Ascarelli-Harrison expression can only be determined with limited precision, which we estimated somewhat subjectively by looking at the variation of the results when different parametrizations of the DD potentials are used: we estimate a relative error on the correction term of approximately $20-30 \%$. As expected, $Z_{A H}$ is significantly worse than the virial expression $Z_{C G}(\rho, \rho)$ : the relative error is $7 \%$ and $12 \%$ for the two values of $\Phi$. We also considered the combination $P^{\prime}(\rho)=\frac{4}{3} P_{C G}(\rho, \rho)-\frac{1}{3} P_{A H}(\rho)$, which is correct to order $\rho^{2}$, see Eq. (46). We find $Z^{\prime}(\rho)=2.43(1), 4.06(2)$ for the two values of $\Phi$. These estimates are slightly closer to the exact result than $Z_{C G}(\rho, \rho)$.

Similar conclusions are reached for the chemical potential. If one uses zero-density po- 
tentials, $\beta \mu^{\operatorname{exc}}(\rho)$ is underestimated by $7 \%$ and $14 \%$ in the two cases, while, by using DD potentials one overestimates the chemical potential by $9 \%$ and $13 \%$. As already discussed in the low-density regime, zero-density and DD potentials are equally inaccurate.

This discussion indicates that, for $\Phi \lesssim 1$, which is the region in which many-body effects are small 45 and therefore CG monoatomic models should work reasonably well, there is little advantage in using DD potentials. Accurate results can only be obtained by using multiblob models. $\underline{43}-\underline{46}, 50,51$ For instance, the tetramer model (model 4MB-2) with zero-density potentials of Ref. 44 gives $Z(\rho)=2.3505(4)$ and $\beta \mu^{\operatorname{exc}}(\rho)=2.57(1)$ for $\Phi=0.8$, in perfect agreement with the full-monomer results. For $\Phi=1.5$, small deviations, of the order of 2-3\%, are found: $Z=3.7901(2)$ and $\beta \mu^{\mathrm{exc}}=5.27(2)$. If more accuracy is needed, one can increase the number of blobs. If one uses a decamer with 10 blobs, $\underline{\underline{44}}$ one obtains for $\Phi=1.5$ the estimates $Z=3.929(3)$ and $\beta \mu^{\mathrm{exc}}=5.3(1)$, which are consistent with the full monomer results.

\section{FUGACITY-DEPENDENT POTENTIALS IN THE GRAND-CANONICAL ENSEMBLE}

The previous discussion applies to the canonical ensemble. However, if one is interested in studying phase coexistence, the grand canonical ensemble is the natural one. In order to implement the CG procedure, we parametrize all thermodynamic variables in terms of the reduced fugacity $z=q_{1} e^{\beta \mu}$, where $q_{1}=Z_{1} / V$ and $Z_{1}$ is the partition function of a single molecule. This guarantees that $z \approx \rho$ for $\rho \rightarrow 0$, both in the original and in the CG model. As before, the basic quantity in the approach is the $X X$ pair correlation function $g(\mathbf{r} ; z)$, which is now a function of the fugacity $z$. If $\rho(z)$ gives the density of the underlying system as a function of $z$, we have obviously $g(\mathbf{r} ; z)=g(\mathbf{r} ; \rho(z))$. The relation with the thermodynamics is provided by the compressibility relation. In the grand canonical ensemble the role of $K$ is played by

$$
H(z)=-z \frac{\partial}{\partial z}\left(\frac{z}{\rho}\right)=\frac{z}{\rho}\left(\frac{\left\langle N^{2}\right\rangle-\langle N\rangle^{2}}{\langle N\rangle}-1\right)
$$

which satisfies ${ }^{33}$

$$
H(z)=z \int d^{3} \mathbf{r}[g(\mathbf{r} ; z)-1]
$$


Using the standard thermodynamic relations we obtain (of course, we assume here that there is no phase transition in the interval $[0, z]$ )

$$
\begin{aligned}
& \rho(z)=z\left[1-\int_{0}^{z} \frac{d w}{w} H(w)\right]^{-1} \\
& \beta P(z)=\int_{0}^{z} \frac{d w}{w} \rho(w)
\end{aligned}
$$

In the CG model one considers a fugacity-dependent (FD) pair potential $V_{F, C G}(\mathbf{r} ; z)$. Again, we should distinguish between the passive and the active approach. For this purpose, we start by considering the fugacity appearing in the potential as a parameter and define

$$
\beta \Omega_{C G}\left(z, z_{p}\right)=-\ln \sum_{N=0}^{\infty} \frac{z^{N}}{N !} Q_{N}\left(z_{p}\right),
$$

where $Q_{N}\left(z_{p}\right)$ is the partition function of a system of $N$ monoatomic molecules interacting via $V_{F, C G}\left(\mathbf{r} ; z_{p}\right)$. The FD potential $V_{F, C G}\left(\mathbf{r} ; z_{p}\right)$ is fixed so that the pair distribution function in the CG model $g_{C G}\left(\mathbf{r} ; z, z_{p}\right)$ for $z_{p}=z$ is equal to the $X X$ pair distribution function in the underlying model. At fixed $z_{p}$, we can define the standard thermodynamic quantities:

$$
\begin{aligned}
& P_{C G}\left(z, z_{p}\right)=-\frac{1}{V} \Omega_{C G}\left(z, z_{p}\right) \\
& \rho_{C G}\left(z, z_{p}\right)=-\frac{\beta z}{V}\left(\frac{\partial \Omega_{C G}}{\partial z}\right)_{T, V, z_{p}} .
\end{aligned}
$$

Since $z_{p}$ is a fixed parameter, the density $\rho_{C G}\left(z, z_{p}\right)$ can be computed as $\langle N\rangle_{z, z_{p}} / V$ as usual [here $\langle\cdot\rangle_{z, z_{p}}$ is the average with respect to the grand canonical distribution defined by Eq. (55)], while $P_{C G}\left(z, z_{p}\right)$ can be determined by using the grand-canonical generalization of the virial expression. If the pair distribution function $g_{C G}\left(\mathbf{r} ; z, z_{p}\right)$ is known, we can also compute thermodynamic quantities using the compressibility route. First, we determine

$$
H_{C G}\left(z, z_{p}\right)=z \int d^{3} \mathbf{r}\left[g_{C G}\left(\mathbf{r} ; z, z_{p}\right)-1\right]
$$

then $\rho_{C G}\left(z, z_{p}\right)$ and $\beta P_{C G}\left(z, z_{p}\right)$ are derived by using Eqs. (53) and (154) at fixed $z_{p}$. The equality of the pair distribution functions implies $H_{C G}(z, z)=H(z)$, but this result does not extend to $\rho_{C G}(z, z)$ and $P_{C G}(z, z)$. For instance,

$$
\begin{aligned}
& \frac{z}{\rho_{C G}(z, z)}=1-\int_{0}^{z} \frac{d w}{w} H_{C G}(w, z) \\
& \quad \neq \frac{z}{\rho(z)}=1-\int_{0}^{z} \frac{d w}{w} H_{C G}(w, w) .
\end{aligned}
$$


Let us now consider the CG model at fugacity $z=z_{p}$. To define the pressure there is no need to distinguish between the active and the passive approach, since $P$ is directly related to $\Omega$. Hence, whatever approach is used, the pressure is always given by $P_{C G}(z, z)$ and therefore always satisfies Clausius theorem. For the density instead, the two approaches give different results. In the passive approach, we should consider $\rho_{C G}(z, z)=\langle N\rangle_{z, z} / V$, while in the active approach we should consider

$$
\begin{aligned}
\rho_{\text {active }}(z) & =-\frac{\beta z}{V} \frac{\partial \Omega_{C G}(z, z)}{\partial z} \\
& =\frac{\langle N\rangle_{z, z}}{V}-\left.\frac{\beta z}{V} \frac{\partial \Omega_{C G}\left(z, z_{p}\right)}{\partial z_{p}}\right|_{z=z_{p}} .
\end{aligned}
$$

This definition looks quite unnatural, since $\rho_{\text {active }}(z)$ differs from the average number of particles per unit volume present in the system.

It is important to stress that the DD potential $V_{D, C G}(\mathbf{r} ; \rho)$ defined in the canonical ensemble and the FD potential $V_{F, C G}(\mathbf{r} ; z)$ are not simply related. If $\rho(z)$ gives the density as a function of $z$ in the underlying model, $V_{F, C G}(\mathbf{r} ; z)$ differs from $V_{D, C G}(\mathbf{r} ; \rho(z))$ : the same thermodynamic state in the microscopic model is represented by different state-dependent potentials, that depend on the ensemble one chooses. Ensemble equivalence does not hold for state-dependent potentials. To understand why the equality does not hold, note that the system at fugacity $z$ interacting with pair potential $V_{F, C G}(\mathbf{r} ; z)$ has density $\rho_{C G}(z, z) \neq \rho(z)$, see Eq. (59). Hence, $V_{F, C G}(\mathbf{r} ; z)$ and $V_{D, C G}(\mathbf{r} ; \rho(z))$ correspond to two systems that have the same pair distribution function but at different densities: hence they cannot be equal. The only exception is the zero-density/zero-fugacity case. Since $\rho_{C G}(0,0)=0$, we have $V_{F, C G}(\mathbf{r} ; z=0)=V_{D, C G}(\mathbf{r} ; \rho=0)$. The ensemble inequivalence, which is completely general and extends to other ensembles, say the isothermal-isobaric one, is clearly related to the thermodynamic inconsistency of state-dependent potentials, which are not able to reproduce simultaneously the correct value of density, chemical potential, and pressure. It is also completely consistent with Henderson's theorem,, 20 which states that, if two systems interacting by pairwise potentials have the same pair distribution function at the same density, then the pair potential is unique. Indeed, in the different ensembles, the pair distribution function is the same, but the density is not. 


\section{COMPARING THE ESTIMATES OF THE DENSITY AND PRESSURE IN THE GRAND CANONICAL ENSEMBLE}

We shall now repeat the analysis of Sec. III in the grand-canonical ensemble, comparing the exact $\rho(z)$ and $P(z)$ with the zero-density approximations $\rho_{C G}(z, 0)$ and $P_{C G}(z, 0)$ and the approximations $\rho_{C G}(z, z)$ and $P_{C G}(z, z)$ obtained by using FD potentials. We will first consider the low-fugacity limit and then we will give results for a specific example, polymers under good-solvent conditions in the semidilute regime.

\section{A. Low-fugacity limit}

Since $g(\mathbf{r} ; z)=g(\mathbf{r} ; \rho(z))$ and we have $z=\rho+O\left(\rho^{2}\right)$ for $\rho \rightarrow 0$, we can reexpress Eq. (24) as

$$
g(\mathbf{r} ; z)=g_{0}(\mathbf{r})+z\left[\hat{g}_{1}(\mathbf{r})+\Delta(\mathbf{r})\right]+O\left(z^{2}\right) .
$$

Using Eq. (52) we obtain

$$
H(z)=z I_{0}+z^{2}\left(I_{0}^{2}+I_{1}+I_{2}\right)+O\left(z^{3}\right)
$$

where $I_{0}, I_{1}$, and $I_{2}$ are defined in Sec. III. Eqs. (53) and (54) give

$$
\begin{gathered}
\rho(z)=z+I_{0} z^{2}+\frac{1}{2} z^{3}\left(3 I_{0}^{2}+I_{1}+I_{2}\right)+O\left(z^{4}\right) \\
\beta P(z)=z+\frac{1}{2} I_{0} z^{2}+\frac{1}{6} z^{3}\left(3 I_{0}^{2}+I_{1}+I_{2}\right)+O\left(z^{4}\right) .
\end{gathered}
$$

Analogously, in the CG model we obtain

$$
H_{C G}\left(z, z_{p}\right)=z I_{0}+z^{2}\left(I_{0}^{2}+I_{1}\right)+z z_{p} I_{2}+O\left(z^{3}, z^{2} z_{p}, z z_{p}^{2}\right)
$$

which satisfies $H_{C G}(z, z)=H(z)$. From this expression we obtain

$$
\begin{gathered}
\rho_{C G}\left(z, z_{p}\right)=z+z^{2} I_{0}+\frac{1}{2} z^{3}\left(3 I_{0}^{2}+I_{1}\right)+z^{2} z_{p} I_{2}, \\
\beta P_{C G}\left(z, z_{p}\right)=z+\frac{1}{2} z^{2} I_{0}+\frac{1}{6} z^{3}\left(3 I_{0}^{2}+I_{1}\right)+\frac{1}{2} z^{2} z_{p} I_{2},
\end{gathered}
$$

disregarding higher-order terms in $z$ and $z_{p}$. For the density in the active approach we obtain instead

$$
\rho_{\text {active }}(z)=z+z^{2} I_{0}+\frac{1}{2} z^{3}\left(3 I_{0}^{2}+I_{1}+3 I_{2}\right)
$$


Comparing the CG results with the exact ones we obtain for the density

$$
\begin{aligned}
\rho_{C G}(z, z)-\rho(z) & =\frac{1}{2} I_{2} z^{3}, \\
\rho_{C G}(z, 0)-\rho(z) & =-\frac{1}{2} I_{2} z^{3}, \\
\rho_{\text {active }}(z)-\rho(z) & =I_{2} z^{3} .
\end{aligned}
$$

In this case $\rho_{C G}(z, 0)$ and $\rho_{C G}(z, z)$ provide approximations that differ by the same amount from the correct, underlying-model result. As in the case of DD potential, the density computed in the active approach is instead significantly worse. If we consider the pressure we obtain

$$
\begin{aligned}
& \beta P_{C G}(z, z)-\beta P(z)=\frac{1}{3} I_{2} z^{3} \\
& \beta P_{C G}(z, 0)-\beta P(z)=-\frac{1}{6} I_{2} z^{3} .
\end{aligned}
$$

It is somewhat surprising that in this case zero-fugacity potentials are more accurate than those that depend on the fugacity. We can also compute the compressibility factor $Z=$ $\beta P / \rho$, obtaining

$$
\begin{aligned}
& Z_{C G}(z, z)-Z(z)=-\frac{1}{6} I_{2} z^{2} \\
& Z_{C G}(z, 0)-Z(z)=\frac{1}{3} I_{2} z^{2} .
\end{aligned}
$$

As in the case of the canonical ensemble, the compressibility factor is instead more accurate for state-dependent potentials.

\section{B. Polymers in the semidilute regime}

We will now repeat in the grand canonical ensemble the computations we have reported in Sec. IIIB. We consider the two values of $z, z \hat{R}_{g}^{3}=2.47$ and 77.7 that correspond to $\Phi=0.8$ and $\Phi=1.5$, the values of the polymer volume fraction considered in Sec. IIIB. To compute the FD potentials - as we already noted they differ from the DD potentials - we use the same technique employed in Ref. 11,12. Since we expect the HNC approximation to be quite accurate ${ }^{33}$ (we verify it below), we relate $z$ and $\rho$ by using the HNC expression $\underline{52,53}$

$$
z=\rho \exp \left[\frac{\rho}{2} \int d^{3} \mathbf{r}\left(h(\mathbf{r})^{2}-h(\mathbf{r}) c(\mathbf{r})-2 c(\mathbf{r})\right)\right],
$$


where $h(\mathbf{r})=g(\mathbf{r} ; z)-1$ and the direct correlation function $c(\mathbf{r})$ is given by the OrnsteinZernike relation $\underline{\underline{33}}$

$$
h(\mathbf{r})=c(\mathbf{r})+\rho \int d^{3} \mathbf{s} c(\mathbf{s}) h(\mathbf{r}-\mathbf{s}) .
$$

Given $z$ and $g(\mathbf{r} ; z)$, we can solve Eqs. (76) and (77) to obtain $\rho$ and $c(\mathbf{r})$. The potential follows from the HNC closure relation:

$$
\beta V_{F, C G}(\mathbf{r} ; z)=h(\mathbf{r})-c(\mathbf{r})-\ln g(\mathbf{r} ; z) .
$$

In this formalism it is easy to understand why the FD potential at, say, $z \hat{R}_{g}^{3}=2.47$ is different from the DD potential at $\Phi=0.8$, although $z \hat{R}_{g}^{3}=2.47$ and $\Phi=0.8$ correspond to the same thermodynamic state of the underlying model. Indeed, Eqs. (76) and (77) cannot be simultaneously satisfied by fixing the chemical potential and the density to the full-monomer values. In the DD case, one fixes $\rho$, obtaining a fugacity that differs from the full-monomer value, while here we fix $z$, obtaining a different density. The HNC procedure can also be applied to other ensembles. For instance, one could obtain the potential in the isobaric ensemble, by simultaneously solving Eqs. (77), (78), and (14), fixing the pressure to its full monomer value. Of course, both $\rho$ and $z$ would differ from the corresponding full-monomer values. The state dependent potentials are reported in Fig. 1 for the two different thermodynamic points of the polymer system we have considered. We also report the potential in the isobaric ensemble, obtained by fixing the pressure. $\underline{54}$ Differences among the potentials are not large on the scale of the figure, but still not negligible. At full overlap $r=0$, we have $\underline{\underline{48}} \beta V_{C G}(r=0)=1.775(5)$ for the zero-density potential (note that this potential is independent of the ensemble). At the state point with $\Phi=0.8$ we have $\beta V_{F, C G}(r=0) \approx 1.902$ for the FD potential and $\beta V_{D, C G}(r=0) \approx 1.955$ for the DD potential. Hence, in the grand canonical ensemble many-body interactions increase repulsion by $8 \%$ with respect to the zero-density case. In the canonical ensemble the effect is $40 \%$ larger: repulsion increases by $11 \%$. At the larger density/fugacity, we have $\beta V_{F, C G}(r=0) \approx 1.955$ and $\beta V_{D, C G}(r=0) \approx 2.030$ for $\mathrm{FD}$ and $\mathrm{DD}$ potentials, respectively: repulsion increases by $11 \%$ and $16 \%$ in the two cases. Note that the observed differences between the DD and FD potentials are of the same order of the difference between the zero-density potential and the state-dependent one, i.e. of the order of the contribution of the many-body interactions, whose inclusion is the main motivation for considering state-dependent potentials.

The HNC procedure gives us estimates of $\Phi$ and of the virial pressure. To check these 

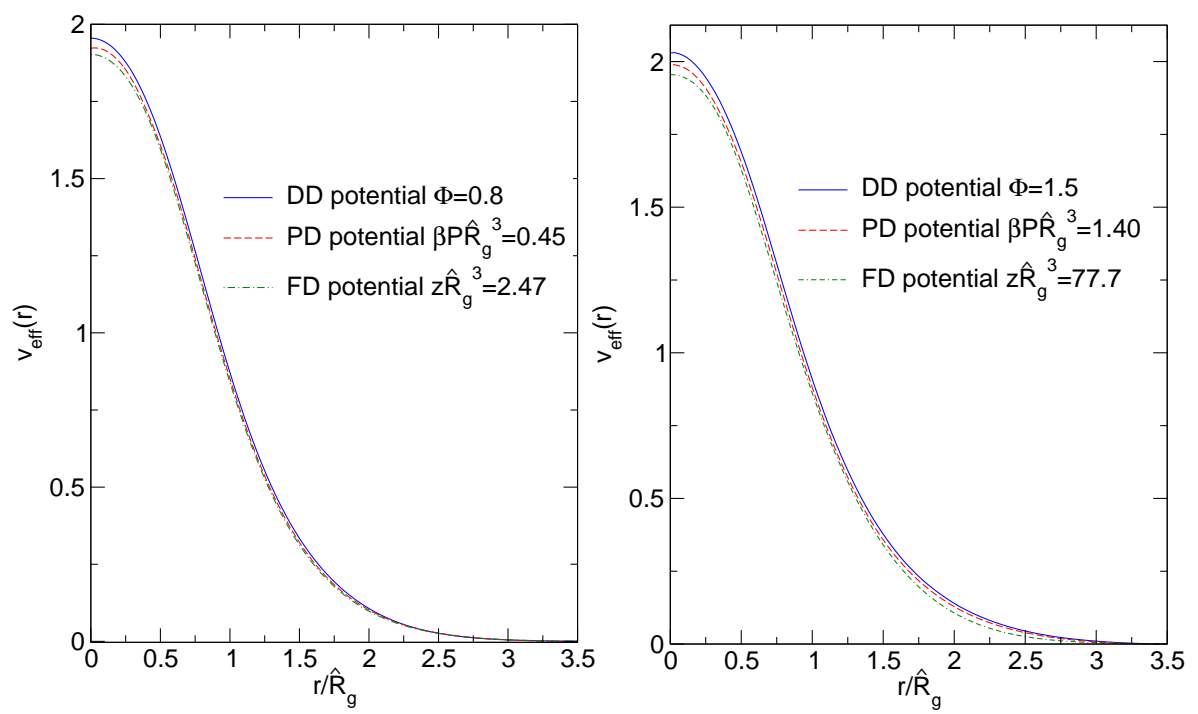

FIG. 1: We report the DD potential (DD), the fugacity dependent (FD) potential, and the pressure dependent (PD) potential as a function of $r / \hat{R}_{g}$. They are obtained by fixing: (left) $\Phi=0.80$, $z \hat{R}_{g}^{3}=2.47, \beta P \hat{R}_{g}^{3}=0.45$ in the three cases, respectively; (right) $\Phi=1.50, z \hat{R}_{g}^{3}=77.7, \beta P \hat{R}_{g}^{3}=$ 1.40 , respectively.

TABLE II: Compressibility factor $Z=\beta P / \rho$ and polymer volume fraction $\Phi=4 \pi \rho \hat{R}_{g}^{3} / 3$ for a polymer CG system at two values of the fugacity. The reference polymer $Z(z)$ and $\Phi(z)$ have been obtain by using the equation of state reported in Ref. 49 .

\begin{tabular}{ccccccc}
\hline \hline$z \hat{R}_{g}^{3}$ & $Z(z)$ & $Z_{C G}(z, 0)$ & $Z_{C G}(z, z)$ & $\Phi(z)$ & $\Phi_{C G}(z, 0)$ & $\Phi_{C G}(z, z)$ \\
\hline 2.47 & 2.35 & $2.2920(1)$ & $2.3338(1)$ & 0.80 & $0.84104(4)$ & $0.77367(4)$ \\
77.7 & 3.90 & $3.7132(2)$ & $3.7819(2)$ & 1.50 & $1.68500(4)$ & $1.47603(4)$ \\
\hline \hline
\end{tabular}

results, we perform grand canonical simulations of the CG model. We measure the density as $\langle N\rangle / V$ and the pressure by using the standard virial expression. The results reported in Table II are in good agreement with those (not reported) obtained by using the HNC approximation, confirming the good accuracy of the procedure.

Let us now compare the results with those of the original, full monomer model. As for the compressibility factor, the FD model gives essentially the correct result for the lowest value of $z$ (the difference is less than 1\%), while it slightly underestimates (by $3 \%$ ) $Z$ for the largest value of $z$. In both cases, results obtained by using FD potentials are more accurate than those obtained by using the corresponding zero-density quantity. The same conclusions 
are reached for $\Phi$, especially for the largest value of $z$. In the analysis of the low-density behavior, we observed that FD potentials are worse than zero-density potentials for what concerns the pressure. Hence, we also consider

$$
\hat{P}=\beta P \hat{R}_{g}^{3}=\frac{3}{4 \pi} Z \Phi
$$

At the smallest value of $z$ we obtain $\hat{P} \approx 0.46020(3)$ by using the zero-density potential and $\hat{P}=0.43105(3)$ by using the FD potential, to be compared with the full-monomer result $\hat{P} \approx 0.45$. The result obtained by using the zero-density potential is closer to the exact, full-monomer result, as expected on the basis of the low-density analysis. At $z \hat{R}_{g}^{3}=77.7$ we have instead $\hat{P} \approx 1.4937(1)$ (zero-density), $\hat{P} \approx 1.3327(1)$ (FD), to be compared with the full-monomer results $\hat{P} \approx 1.40$. The state-dependent potential provides now a result which is slightly more accurate.

From these results, it is clear that state-dependent potentials provide only approximations to pressure and density that worsen as the fugacity increases. Accurate results can only be obtained by using CG multiblob models. If we use the tetramer model of Ref. 44, for $z \hat{R}_{g}^{3}=2.47$ we have $Z=2.3457(5)$ and $\Phi=0.7978(1)$, while for $z \hat{R}_{g}^{3}=77.7$ we have $Z=3.8450(7)$ and $\Phi=1.5257(1)$. Good agreement is observed for the lowest value of the fugacity, while small discrepancies occur for the second one. If we use the decamer model 14 we would obtain for $z \hat{R}_{g}^{3}=77.7$ the estimates $Z=3.925(1)$ and $\Phi=1.4984(3)$, which are now fully consistent with the full monomer results.

\section{CONCLUSIONS}

In this paper we have considered structurally derived state-dependent potentials for CG models, with the purpose of understanding how useful they are in predicting the thermodynamics of complex systems. As already discussed in Ref. 38, the virial or the AscarelliHarrison ${ }^{41}$ pressure computed in the CG model do not reproduce the pressure of the underlying system. But the same conclusions apply to essentially all thermodynamic quantities. In the canonical ensemble Widom's method does not give the correct underlying-model chemical potential, while in the grand canonical ensemble the virial pressure or $\langle N\rangle / V$ do not reproduce pressure and density of the underlying system. It is clear from the discussion that these results are not specific to these quantities. If the pair distribution function is 
used to define the state-dependent potentials, only the quantities that are directly related to the pair distribution function, like $K(\rho)$ or $H(z)$, are identical in the $\mathrm{CG}$ and in the underlying model. All others differ. From a practical point of view, since state-dependent interactions are commonly used in large-scale simulations, it is important to quantify the differences between the CG estimates of the thermodynamic quantities and the underlying model values. In all cases we find that the passive approach provides the most accurate results. This is quite pleasant since the active-approach definitions look quite unnatural: the pressure defined in the canonical ensemble does not satisfy Clausius' virial theorem-hence the pressure is not directly related to the average force on the boundaries - while the density in the grand canonical ensemble defined by differentiating the grand potential differs from $\langle N\rangle / V$.

Ref. 38 noted that the pressure of the underlying model can be obtained by using the compressibility route. However, it should be clear that one is referring to the compressibility route in the underlying model, i.e., to Eq. (4). But this means that one is obtaining the pressure from a quantity computed in the underlying microscopic model, hence the DD potential plays no role. Analogously, in the grand-canonical ensemble, the correct density can be obtained by integrating $H(z)$, but again this calculation does not really make use of the model with FD potentials.

It is important to stress that structurally derived state-dependent potentials depend on the ensemble one uses, or rather on the thermodynamic variable one uses to identify the thermodynamic state of the system. At a given thermodynamic state of the microscopic system, DD potentials and FD potentials differ, since they correspond to CG monoatomic systems that have the same pair distribution function but different densities and chemical potentials. Therefore, DD canonical potentials should not be used to predict phase lines, since the coexistence analysis is typically performed in other ensembles.

Although in this paper we have only discussed DD and FD potentials, the results are general and apply to other ensembles, for instance to pressure dependent potentials in the isothermal-isobaric ensemble. However, the conclusions do not necessarily apply to potentials that are only temperature dependent. If the thermodynamic state one is considering can be connected by a thermodynamic path at fixed temperature to the zero-density state (this is always true in the absence of phase transitions or at least in the low-density phase), since temperature does not appear explicitly in the integrations leading to the free ener- 
gies, the free energy is essentially correct (one should only add the zero-density contribution of a single, isolated molecule). Hence, all thermodynamic quantities that can be obtained from the free energies are correct. An interesting method for transferring CG potentials between temperatures in the context of the multiscale coarse-graining force-matching method is described in Ref. 55. It is important to note that the ensemble dependence does not occur in CG models defined by using the multiscale coarse-graining approach based on the force-matching method. $\frac{15}{}$ Indeed, the distribution of the atomistic configurations is obviously ensemble independent in the infinite-volume limit, hence also the matching conditions and the CG model do not depend on the ensemble (see Ref. 56 for a discussion in the isobaric-isothermal ensemble).

Our results also shed some light on mixed approaches in which one matches the pair distribution and some other thermodynamic property. These approaches are conceptually correct only if the thermodynamic quantity is appropriately chosen. In the canonical ensemble, the only correct choice is the isothermal compressibility, i.e. the quantity $K$ we have introduced, while in the grand canonical ensemble one should use $H(z)$. It is also possible to enforce the value of the pressure, i.e. to work in the isothermal-isobaric ensemble, but in this case one should relax the condition that the microscopic system and the CG model have the same density. For each choice, a different state-dependent potential is obtained, as observed, for instance, in Ref. 57 in the context of CG models of water. Note that these observations can be trivially generalized to multicomponent systems. In this case, one can analogously enforce the Kirkwood-Buff 58 equations that relate thermodynamic properties to integrals of distribution functions, see Ref. 59 for a recent example.

The fact that DD potentials are unable to provide the correct results for the thermodynamic quantities should not be surprising. After all, even with the use of a state-dependent potential, the CG model is still an approximation to the original microscopic model. Hence, one should consider this approach as a simple method to obtain relatively good approximations for the behavior of complex systems.

It is easy to find situations, both in theoretical or experimental work, in which statedependent potentials are useful. For instance, one could consider a system of complex molecules for which simulations are particularly difficult. In order to obtain with reasonable precision the value of the thermodynamic quantities in a range of densities, one could perform a simulation at a density $\rho_{p}$, measuring all thermodynamic quantities and the density- 
dependent potential $V_{D, C G}\left(\mathbf{r} ; \rho_{p}\right)$. Then, to compute the pressure for $\rho \neq \rho_{p}$, one could write

$$
P(\rho)=P\left(\rho_{p}\right)+\left[P(\rho)-P\left(\rho_{p}\right)\right]
$$

and determine the second term by using the CG model that uses the DD potential computed at density $\rho_{p}$. Hence, one could estimate

$$
P(\rho)=P\left(\rho_{p}\right)+\left[P_{C G}\left(\rho, \rho_{p}\right)-P_{C G}\left(\rho_{p}, \rho_{p}\right)\right],
$$

using the $\mathrm{CG}$ model to compute the differences between the state point of interest and that for which exact results, i.e. results obtained in the microscopic model, are available. CG models with zero-density potentials use exactly this strategy, fixing $\rho_{p}=0$. The same strategy could also be used in experiments, allowing one to obtain all thermodynamic informations in a range of densities, using experimentally determined thermodynamic and structural data at $\rho_{p}$.

We have checked this strategy in our polymer example. Assuming that the exact results for $P$ and $\mu$ are available at $\Phi=0.8$, we wish to compute $P$ and $\mu$ at $\Phi=1.5$. Using the DD potential computed at $\Phi=0.8$, we obtain $Z=2.44790(4)$ and $\beta \mu^{\text {exc }}=2.8108(3)$ at

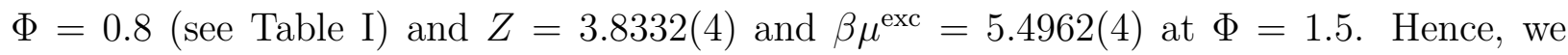
would estimate at $\Phi=1.5$

$$
Z=3.74 \quad \beta \mu^{\mathrm{exc}}=5.25,
$$

to be compared with the FM predictions $Z \approx 3.90$ and $\beta \mu^{\text {exc }}=5.38$. Estimates (82) are significantly more precise than those reported in Table I, confirming the usefulness of the approach.

Finally, note that we have considered here very simple CG models in which the CG molecules are monoatomic. In practical soft-matter applications CG models are usually polyatomic. It is quite obvious that the same conclusions apply to these more complex models: also in this case structurally derived potentials depend on the ensemble and thermodynamics is only approximately reproduced.

\section{Acknowledgments}

The authors thank Barbara Capone and Luigi Delle Site for useful comments. C.P. is supported by the Italian Institute of Technology (IIT) under the SEED project grant number 
259 SIMBEDD Advanced Computational Methods for Biophysics, Drug Design and Energy Research.

† Electronic address: giuseppe.dadamo@aquila.infn.it

$\ddagger$ Electronic address: andrea.pelissetto@roma1.infn.it

$\S$ Electronic address: carlo.pierleoni@aquila.infn.it

1 G. A. Voth, ed., Coarse-Graining of Condensed Phases and Biomolecular Systems (CRC Press, Boca Raton, 2009).

2 R. Feller, Guest Editor, Phys. Chem. Chem. Phys. 11, 1853 (2009).

3 M. Wilson, Guest Editor, Soft Matter 5, 4341 (2009).

4 Multiscale Modelling of Soft Matter, Faraday Discussion 144, 1 (2010).

5 L. Larini, L. Lu, and G. A. Voth, J. Chem. Phys. 132, 164107 (2010).

6 G. S. Rushbrooke and M. Silbert, Mol. Phys. 12, 505 (1967).

7 J. S. Rowlinson, Mol. Phys. 12, 513 (1967).

8 J. A. Barker, D. Henderson, and W. R. Smith, Mol. Phys. 17, 579 (1969).

9 G. Casanova, R. J. Dulla, D. A. Jonah, J. S. Rowlinson, and G. Saville, Mol. Phys. 18, 589 (1970).

10 M. A. van der Hoef and P. A. Madden, J. Chem. Phys.111, 1520 (1999).

11 A. A. Louis, P. G. Bolhuis, J. P. Hansen, and E. J. Meijer, Phys. Rev. Lett. 85, 2522 (2000);

P. G. Bolhuis, A. A. Louis, J. P. Hansen, and E. J. Meijer, J. Chem. Phys. 1144296 (2001).

12 P. G. Bolhuis and A. A. Louis, Macromolecules 35, 1860 (2002).

13 F. Müller-Plathe, Chem. Phys. Chem. 3, 754 (2002).

14 C. Peter and K. Kremer, Soft Matter 5, 4357 (2009).

15 S. Izvekov and G. A. Voth, J. Phys. Chem B 109, 2469 (2005); J. Chem. Phys. 123, 134105 (2005).

16 W. G. Noid, J.-W. Chu, G. S. Ayton, V. Krishna, S. Izvekov, G. A. Voth, A. Das, and H. C. Andersen, J. Chem. Phys. 128, 244114 (2008); W. G. Noid, P. Liu, Y. Wang, J.-W. Chu, G. S. Ayton, S. Izvekov, H. C. Andersen, and G. A. Voth, J. Chem. Phys. 128, 244115 (2008).

17 W. G. Noid, J. W. Chu, G. S. Ayton, and G. A. Voth, J. Phys. Chem. B 111, 4116 (2007); J. W. Mullinax and W. G. Noid, Phys. Rev. Lett. 103, 198104 (2009). 
18 S. Merabia and I. Pagonabarraga, J. Chem. Phys. 127, 054903 (2007).

19 E. C. Allen and G. C. Rutledge, J. Chem. Phys. 130, 034904 (2009).

20 R. L. Henderson, Phys. Lett. 49A, 197 (1974).

21 B. M. Axilrod and E. Teller, J. Chem. Phys. 11, 299 (1943).

22 C. N. Likos, Phys. Rep. 348, 267 (2001); Soft Matter 2, 478 (2006).

23 C. N. Likos, H. Löwen, M. Watzlawek, B. Abbas, O. Jucknischke, J. Allgaier, and D. Richter, Phys. Rev. Lett. 80, 4450 (1998).

24 M. Watzlawek, C. N. Likos, and H. Löwen, Phys. Rev. Lett. 82, 5289 (1999).

25 A. Jusufi, J. Dzubiella, C. N. Likos, C. von Ferber, and H. Löwen, J. Phys.: Condens. Matter 13, 6177 (2001).

26 J. Dzubiella, C. N. Likos, and H. Löwen, J. Chem. Phys. 116, 9518 (2002).

27 G. D'Adamo, A. Pelissetto, and C. Pierleoni, J. Chem. Phys. 136, 224905 (2012).

28 D. Reith, M. Pütz, and F. Müller-Plathe, J. Comput. Chem. 24, 1624 (2003).

29 A. Lyubartsev and A. Laaksonen, Phys. Rev. E 52, 3730 (1995).

30 A. Soper, Chem. Phys. 202, 295 (1996).

31 H. Meyer, O. Biermann, E. Faller, D. Reith, and F. Müller-Plathe, J. Chem. Phys. 113, 6264 (2000).

32 R. L. C. Akkermans and W. J. Briels, J. Chem. Phys. 114, 1020 (2001).

33 J. P. Hansen and I. McDonald, Theory of Simple Liquids, 3rd ed. (Academic Press, Amsterdam, 2006)

34 M. S. Shell, J. Chem. Phys. 129, 144108 (2008).

35 V. Krishna and L. Larini, J. Chem. Phys. 135, 124103 (2011).

36 V. Rühle, C. Junghans, A. Lukyanov, K. Kremer, and D. Andrienko, J. Chem. Theory Comput. 5, 3211 (2009).

37 F. H. Stillinger, H. Sakai, and S. Torquato, J. Chem. Phys. 117, 288 (2002).

38 A. A. Louis, J. Phys.: Condens. Matter 14, 9187 (2002).

39 M. E. Johnson, T. Head-Gordon, and A. A. Louis, J. Chem. Phys. 126, 144509 (2007).

40 C. F. Tejero and M. Baus, J. Chem. Phys. 118, 892 (2003); C. F. Tejero and E. Lomba, Mol. Phys. 107, 349 (2009).

41 P. Ascarelli and R. Harrison, Phys. Rev. Lett. 22, 285 (1969).

42 B. Widom, J. Chem. Phys. 39, 2802 (1963). 
43 G. D'Adamo, A. Pelissetto, and C. Pierleoni, Soft Matter 8, 5151 (2012).

44 G. D’Adamo, A. Pelissetto, and C. Pierleoni, J. Chem. Phys. 137, 024901 (2012).

45 C. Pierleoni, B. Capone, and J. P. Hansen, J. Chem. Phys. 127, 171102 (2007).

46 T. Vettorel, G. Besold, and K. Kremer, Soft Matter 6, 2282 (2010).

47 As in our previous work, we determine $g(\mathbf{r} ; \rho)$ by using the Domb-Joyce model at $w=0.505838$, see Ref. 60 for a precise definition of the model, simulating lattice chains of length 2400 . As discussed in Ref. 60 this should provide an accurate estimate of the pair distribution function in the asymptotic (infinite degree of polymerization), universal limit.

48 A. Pelissetto and J.-P. Hansen, J. Chem. Phys. 122, 134904 (2005).

49 A. Pelissetto, J. Chem. Phys. 129, 044901 (2008).

50 D. Fritz, V. A. Harmandaris, K. Kremer, and N. F. A. van der Vegt, Macromolecules 42, 7579 (2009).

51 A. J. Clark and M. G. Guenza, J. Chem. Phys. 132, 044902 (2010); A. J. Clark, J. McCarty, I. Y. Lyubimov, and M. G. Guenza, Phys. Rev. Lett. 109, 168301 (2012).

52 T. Morita, Prog. Theor. Phys. 23, 839 (1960).

53 P. Attard, J. Chem. Phys. 94, 2370 (1991).

54 In the full-monomer model we have $\beta P \hat{R}_{g}^{3}=0.45$ and $\beta P \hat{R}_{g}^{3}=1.40$ for $\Phi=0.80$ and 1.50 . For comparison, we report the pressure-dependent potentials for these two values of the pressure. Using the HNC approximation we can also compute the thermodynamic variables using these state-dependent interactions. We obtain $\Phi=0.784, Z=2.397, \beta \mu^{\text {exc }}=2.72$ for $\beta P \hat{R}_{g}^{3}=0.45$ and $\Phi=1.484, Z=3.942, \beta \mu^{\mathrm{exc}}=5.71$ for $\beta P \hat{R}_{g}^{3}=1.40$.

55 V. Krishna, W. G. Noid, and G. A. Voth, J. Chem. Phys. 131, 024103 (2009).

56 A. Das and H. C. Andersen, J. Chem Phys. 132, 164106 (2010).

57 H. Wang, C. Junghans, and K. Kremer, Eur. Phys. J. E 28, 221 (2009).

58 J. G. Kirkwood and F. P. Buff, J. Chem. Phys. 19, 774 (1951).

59 P. Ganguly, D. Mukherji, C. Junghans, and N. F. A. van der Vegt, J. Chem. Theory Comput. 8, $1802(2012)$.

60 S. Caracciolo, B. M. Mognetti, and A. Pelissetto, J. Chem. Phys. 125, 094903 (2006). 